\title{
Reconstruction of Jet Properties in Pb-Pb Collisions with the ALICE-Experiment
}

\author{
C. Klein-Bösing for the ALICE Collaboration \\ Institut für Kernphysik - Münster, Germany \\ ExtreMe Matter Institute, GSI - Darmstadt, Germany
}

\begin{abstract}
.
The study of the hot and dense medium created in heavy-ion collisions via the effect of parton energy loss is one of the major goals of jet and high- $p_{\mathrm{t}}$ measurements in these reactions. In particular the modifications of the longitudinal and transverse jet structure. Here the subtraction of the underlying heavy-ion event, as well as a precise knowledge of the background induced fluctuations of the reconstructed jet energy is required. We extract the modification of the near- and away-side jet yield in heavy-ion collisions at the LHC and present a detailed assessment of background fluctuations for jet reconstruction.
\end{abstract}

Keywords: Heavy-ion collisions, Quark-Gluon Plasma,Hard scattering, Jet quenching, Correlations PACS: $13.85 .-\mathrm{t}, 25.75 . \mathrm{Gz}, 25.75 . \mathrm{Bh}$

\section{INTRODUCTION}

Hard scattered partons in heavy-ion collisions provide a well defined probe for the complete evolution of the medium created in heavy-ion collisions since the scattering occurs well before the medium is formed $(t \approx 1 / Q \ll 1 \mathrm{fm} / c)$. Thus, the modification of the final state fragmentation process of the parton compared to the fragmentation into the QCD vacuum in pp allows to map out the properties of the QCD medium [1,2]. Here, the most direct access to the original parton properties is given by the full reconstruction of jets. Already the first measurements at LHC revealed a striking energy imbalance between back-to-back dijets [3], pointing to a significant dissipation of jet energy in the medium. A quantitative understanding of these results requires a precise understanding of background-induced fluctuations of the measured jet energy, which can distort the energy balance even in the absence of any other medium effects [4]. It is also important to corroborate full jet recoqnstruction measurements, with jet properties extracted from single particle [5] and correlation measurements, to take advantage of a direct fragmentation bias and a different susceptibility to the underlying event background. In case of the correlation measurement, this provides access to jet properties in a momentum range. where full jet reconstruction is dominated by background fluctuations.

\section{HARD PROBES WITH THE ALICE-EXPERIMENT}

The presented data were collected by the ALICE experiment [6] during the first heavy-ion run of the LHC in the fall of 2010 with lead ions colliding at an energy of $\sqrt{s_{\mathrm{NN}}}=2.76 \mathrm{TeV}$. The analyses are based on tracks of charged particles reconstructed in the Time-Projection-Chamber (TPC) together with vertexing information from the Inner Tracking System (ITS). This ensures maximum azimuthal angle $(\phi)$ uniformity of reconstructed tracks with transverse momenta down to $p_{\mathrm{t}}=150 \mathrm{MeV} / c$.

\section{Triggered Particle Correlations}

In this approach a trigger particle with $p_{\mathrm{t}}>p_{\mathrm{t}, \text { trig }}$ is used to define the direction for the measurement of azimuth

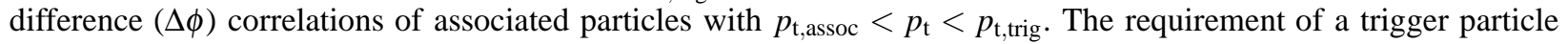
prefers the selection of unquenched jets close to the surface at the near-side, while the away-side jets have to traverse a longer path through the medium. At lower $p_{\mathrm{t} \text {,trig }}$ where particle production is not dominated by jet fragmentation, collective effects [7], have a strong influence on the $\Delta \phi$ distributions and a Fourier-decomposition can be used to 

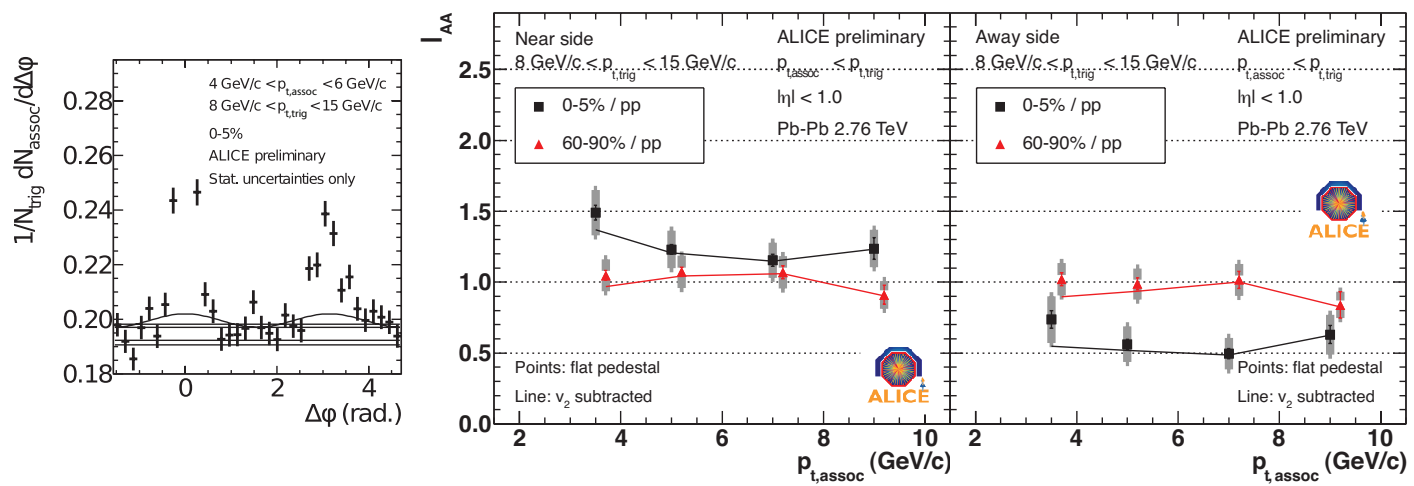

FIGURE 1. Left: Per-trigger yield (normalized by $(\Delta \eta=1.6)$ in an example bin (zoom, the near-side peak is off scale). The determined pedestal values (horizontal lines) and the $v_{2}$ component $(\cos 2 \Delta \phi)$ are shown. Right: $I_{A A}$ for near- and away-side in central and peripheral collisions: the data points are calculated with a flat pedestal; the line is based on $v_{2}$ subtracted yields.

quantify the role of long range correlations (see e.g. [8]). Here, the trigger particle $p_{\mathrm{t}}$ has been chosen such that collective effects on the $\Delta \phi$ distribution are small and jet-like correlations dominate $\left(p_{\mathrm{t} \text {,trig }}>8 \mathrm{GeV} / c, p_{\mathrm{t} \text {,assoc }}>\right.$ $3 \mathrm{GeV} / \mathrm{c}$ ). The distribution of associated particles with respect to the $\phi$ direction of the trigger particle is normalized to the number of triggers as illustrated in Figure 1, where a clear back-to-back structure at a distance $\Delta \phi=\pi$ is seen. As illustrated in Figure 1, the background (i.e. non-jet) contribution to the raw trigger yield is subtracted using different assumptions on the magnitude of a constant pedestal or a constant pedestal plus the expected contribution from elliptic flow, which leads to an additional $\cos (2 \Delta \phi)$ variation. After background subtraction the per-trigger-yield $Y$ is obtained by integrating the near- and away-side peak, respectively. To quantify the modification of the jet fragmentation process in different bins of $p_{\mathrm{t} \text {,assoc }}$, the ratio $I_{A A}=Y_{P b-P b} / Y_{p p}$ to the yield measured in pp at $\sqrt{s}=2.76 \mathrm{TeV}$ is used. As seen in Figure 1, for peripheral collisions (60-90\%) neither the yield on the away-side, nor on the near-side is modified compared to elementary reactions. However, for central (0-5\%) a significant suppression of the away-side yield is observed for all bins of $p_{\mathrm{t} \text {,assoc }}$ as expected from parton energy loss. Furthermore, the yield at the near-side is enhanced, which can be interpreted as a consequence of jet quenching as well. One effect is that near-side gluons contribute less to the fragmentation yield, since they are more effectively quenched in the medium, due to their larger color charge. Quarks fragment harder and thus can give rise to the enhanced yield [9].

\section{Jet Background Characterization}

The separation of jet properties from the global event characteristics, in the case of triggered correlation achieved via a large $p_{t, \text { trig }}$ becomes more challenging in the case of full jet reconstruction. Here, the goal is to recover the full parton momentum by the use of various algorithms which cluster the final state particles to a jet (here the sequential recombination algorithms $k_{\mathrm{t}}$ and anti- $k_{\mathrm{t}}$ from the FastJet package are employed [10]). From the jet momentum and the particles within the jet in principle the fragmentation function of the parton and its medium modification can be reconstructed. However, in the context of heavy-ion collisions one has to take into account the significant contribution of the underlying event to the reconstructed jet momentum, which can be reduced e.g. by a $p_{\mathrm{t}}$ or energy threshold of the input particles to the jet finders and by a reduced distance parameter $R$. All of these introduce additional biases on the fragmentation pattern of the reconstructed jets and the remaining background has to be subtracted from the reconstructed jet $p_{\mathrm{t}}$. For the present analysis the background subtraction scheme proposed in [11] is employed, which uses an event-by-event median of the background momentum per unit area $\rho$, which accounts for the expected contribution in a jet with area $A$. In the following the fluctuations induced by correlated and uncorrelated variations of the background density within one event are investigated. This measurement is of particular importance for the correction of the inclusive jet spectrum and for the quantitative interpretation of jet momentum imbalance. The current study is limited to the charged component of the jet, and will be extended in the next $\mathrm{Pb}-\mathrm{Pb}$ run by ALICE with the addition of the EMCAL to the combined charged and neutral particle information. 


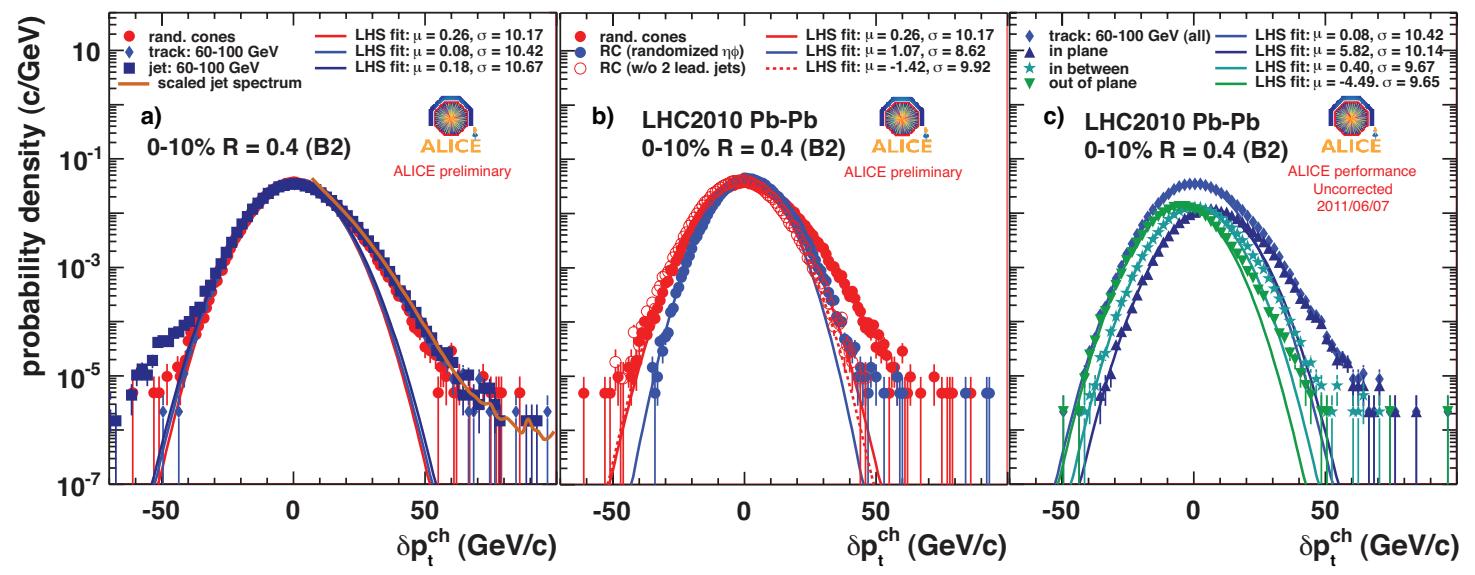

FIGURE 2. $\delta p_{\mathrm{t}}$ distributions for reconstructed charged particles with $p_{\mathrm{t}}>150 \mathrm{MeV} / c$ and different probes types. The lefthand-side of the distributions is fit to a Gaussian that is plotted over the full range to indicate the deviation from the Gaussian shape.

The fluctuations are quantified by embedding well defined probes into the measured heavy-ion events and calculating the residuals after background correction $\delta p_{\mathrm{t}}=p_{\mathrm{t}}^{\text {rec }}-\rho A-p_{\mathrm{t}}^{\text {probe }}$. The different probes involve: (i) random cones (RC) of fixed area placed into the acceptance, (ii) high- $p_{\mathrm{t}}$ particles acting as effective seeds for jet finding at this position (iii) pp jets from a full PYTHIA+GEANT simulation. The different cases are shown in Figure 2a) for the $0-10 \%$ most central events. They are centered at zero, showing the validity of our background subtraction scheme and show in general a good agreement amongst each other. The deviations from a purely Gaussian shape is clearly seen, the width of the Gaussian is in all cases larger than $10 \mathrm{GeV} / c$. One source of the deviation is the presence of jets in the event: this can enhance the local $p_{\mathrm{t}}$ density in the rare case of a hard scattering significantly. In Figure $1 \mathrm{~b}$ ), random cones are embedded into real events for two additional cases: with a distance of $D>1$ in the $\eta \phi$-plane to the two leading jets and after randomizing the momentum vectors of the event in the acceptance. In both cases the tail on the right-hand-side attributed to jets is much reduced. In the latter case also the width on the left side is reduced, pointing to correlated fluctuation not originating from jets. These can be explained by the presence of collective flow, which modulates the particle density and the average momentum in a heavy-ion event depending on the direction to the reaction plane (given by the beam direction and the impact parameter of the colliding nuclei). Figure 1c) illustrated this with the shift of the $\delta p_{\mathrm{t}}$ distribution resulting from an over and under-subtraction of the background, when embedding into different direction with respect to the reaction plane.

\section{SUMMARY}

We presented the modification of the near- and away-side jet yield measured via triggered hadron correlations in heavy-ion collisions at the LHC and also showed an assessment of the background fluctuations for jet reconstruction in heavy-ion collisions, which exceed $10 \mathrm{GeV} / c$ for charged particles in central $\mathrm{Pb}-\mathrm{Pb}$ reactions.

\section{ACKNOWLEDGMENTS}

Copyright CERN for the benefit of the ALICE Collaboration.

\section{REFERENCES}

1. C. A. Salgado, and U. A. Wiedemann, Nucl. Phys. A715, 783-786 (2003), hep-ph/ 0209025.

2. B. Alessandro, et al., J. Phys. G32, 1295-2040 (2006).

3. G. Aad, et al., Phys.Rev.Lett. 105, 252303 (2010), 1011.6182. 
4. M. Cacciari, G. P. Salam, and G. Soyez, Eur. Phys. J. C71, 1692 (2011), 1101.2878.

5. K. Aamodt, et al., Phys. Lett. B696, 30-39 (2011), 1012.1004.

6. K. Aamodt, et al., JINST 0803, S08002 (2008).

7. K. Aamodt, et al., Phys. Rev. Lett. 105, 252302 (2010), 1011.3914.

8. J. F. Grosse-Oetringhaus (2011), 1107.0556.

9. T. Renk, and K. Eskola (2011), 1106.1740.

10. M. Cacciari, and G. P. Salam, Phys. Lett. B641, 57-61 (2006), hep-ph/ 0512210.

11. M. Cacciari, and G. P. Salam, Phys. Lett. B659, 119-126 (2008), 0707 . 1378. 